\title{
Método Iterativo Baseado em LMIs para Estabilização por Realimentação de Saída de Sistemas Lineares Incertos Positivos Contínuos no Tempo
}

\author{
Álvaro A. Lemaire * Cecília F. Morais * Ricardo C. L. F. Oliveira * \\ Pedro L. D. Peres* \\ ${ }^{*}$ Faculdade de Engenharia Elétrica e de Computação, \\ Universidade Estadual de Campinas - UNICAMP \\ Av. Albert Einstein, 400, 13083-852, Campinas, SP, Brasil \\ (e-mail: \{arnanz, cfmorais, ricfow, peres\}@dt.fee.unicamp.br).
}

\begin{abstract}
This paper proposes a new approach for static output-feedback control of continuoustime positive uncertain linear systems. The stabilizing controller is obtained by means of an iterative procedure based on linear matrix inequalities. In the synthesis conditions, the control gain is dealt with as an optimization variable, without changes of variables or structure constraints in the Lyapunov matrix that certifies the closed-loop stability of the system. Versatile, the method can be applied in the case of both uncertain and precisely known systems, considering output or state-feedback. Examples from the literature, comparisons and statistical tests illustrate the results.

Resumo: Este artigo propõe uma nova abordagem para o controle por realimentação estática de saída de sistemas lineares incertos positivos contínuos no tempo. O controlador estabilizante é obtido por meio de um procedimento iterativo baseado em desigualdades matriciais lineares. Nas condições de síntese, o ganho de controle é tratado diretamente como variável de otimização, sem mudanças de variáveis ou imposições de restrições de estrutura na matriz de Lyapunov que certifica a estabilidade do sistema em malha fechada. Versátil, o método pode ser aplicado tanto no caso de sistemas incertos quanto precisamente conhecidos, considerando realimentação de saída ou de estados. Exemplos da literatura, comparações e testes estatísticos ilustram os resultados.
\end{abstract}

Keywords: Robust Control; Output-feedback; Positive Uncertain Linear Systems; Continuous-time Systems; Linear Matrix Inequalities.

Palavras-chaves: Controle Robusto; Realimentação de Saída; Sistemas Lineares Positivos Incertos; Sistemas Contínuos no Tempo; Desigualdades Matriciais Lineares.

\section{INTRODUÇÃO}

Devido à evolução dos procedimentos numéricos e ao grande avanço da capacidade de processamento computacional, problemas de controle de sistemas dinâmicos sujeitos a incertezas, cada vez mais complexos, começaram a ser tratados. Nesse cenário, destacam-se as técnicas de otimização baseadas em programação semidefinida, sobretudo as formuladas em termos de desigualdades matriciais lineares (em inglês, Linear Matrix Inequalities - LMIs). Veja Boyd et al. (1994), El Ghaoui and Niculescu (2000) e Oliveira and Peres (2010) para uma introdução ao tema.

Adicionalmente, uma ampla gama de problemas práticos apresenta uma característica importante: a não-negatividade dos estados e das saídas, geralmente variáveis associadas a grandezas físicas que só assumem valores positivos ou nulos. Tais sistemas são conhecidos como sistemas positivos. Dentre as princi-

\footnotetext{
ऋ O presente trabalho foi realizado com apoio da Coordenação de Aperfeiçoamento de Pessoal de Nível Superior - Brasil (CAPES) - Código de Financiamento 001, CNPq, FAPESP (Processo 2017/18785-5) e Projeto de P\&D PD0063-3047/2018 - PA3047 da CPFL Energia.
}

pais aplicações dessa classe de sistemas em engenharia, podem ser citados os processos industriais envolvendo reatores químicos, trocadores de calor, redes de reservatórios, fluxo em redes ou sistemas de armazenamento ou comunicações (Berman and Plemmons, 1979; Farina and Rinaldi, 2000; Luenberger, 1979). Fora da área de engenharia, existem outras aplicações como na economia e na sociologia, com modelos demográficos e sociológicos sobre populações, ou na medicina e na biologia, analisando e controlando cultivos de bactérias ou células (Berman and Plemmons, 1979; Farina and Rinaldi, 2000; Luenberger, 1979).

O estudo de sistemas positivos é uma área ainda aberta à pesquisa, pois nem todos os métodos empregados no tratamento de sistemas lineares podem ser estendidos diretamente para sistemas positivos, uma vez que esses estão definidos em cones convexos e não em um espaço linear (Caccetta and Rumchev, 2000). Assim, este artigo dedica-se à síntese de controladores para sistemas lineares positivos contínuos no tempo. Note que, para que o sistema em malha fechada seja positivo, é necessário (adicionalmente ao exigido no problema de controle clássico) garantir que a matriz dinâmica do sistema em malha fechada seja Metzler (ou seja, com elementos não negativos fora da 
diagonal). Várias abordagens têm surgido na literatura com o objetivo de tratar esse problema (Briat, 2013; Ebihara et al., 2014; Shen and Lam, 2015, 2017, 2016; Tanaka and Langbort, 2011; Wang and Huang, 2013). Pode-se dizer que um dos desafios do controle de sistemas positivos é estender métodos que funcionam bem para sistemas com uma entrada de controle e uma saída, por exemplo, baseados em programação linear (Arneson and Langbort, 2012; Ait Rami, 2011; Roszak and Davison, 2009), para o caso multivariável. Embora condições de síntese de controle por realimentação de estados para sistemas positivos por meio de LMIs possam ser obtidas como uma extensão direta dos métodos apresentados na literatura para sistemas lineares tradicionais, a dificuldade maior está em tratar a restrição de que o sistema em malha fechada mantenha-se positivo, o que pode ser conseguido impondo-se uma estrutura diagonal à matriz utilizada para computar o ganho, como foi feito em Tanaka and Langbort (2011); Ebihara et al. (2014). Outra alternativa poderia ser, por exemplo, o uso de abordagens iterativas que trabalham diretamente com as variáveis do ganho (Felipe, 2017), estratégias de cômputo do ganho em dois estágios (Agulhari et al., 2012; Peaucelle and Arzelier, 2001) ou LMIs com variáveis escalares (Vieira et al., 2015), nas quais pode-se impor restrições às variáveis de recuperação do ganho.

A estratégia adotada neste artigo faz o uso de procedimentos iterativos baseados em LMIs. Para isso, é elaborada uma condição em termos de LMIs dependentes de parâmetros, baseada em Felipe (2017), para síntese de controladores estabilizantes por realimentação de estados ou estática de saída, na qual a matriz dinâmica do sistema não aparece multiplicando nenhuma variável de decisão e o ganho estabilizante de controle é tratado diretamente como uma variável de otimização do problema. Sendo assim, o conservadorismo que existe nos outros métodos da literatura, na forma de LMIs, adaptados para síntese de controladores para sistemas contínuos positivos (imposição de estrutura diagonal na matriz de Lyapunov ou na matriz quadrada de recuperação do ganho) desaparece. Como resultado, o método proposto, em tese, pode fornecer resultados menos conservadores. Uma novidade com relação à maioria das técnicas de controle de sistemas por realimentação de saída é que a matriz $C(\alpha)$ pode ter qualquer estrutura, por exemplo, pode ser politópica, não havendo necessidade da aplicação de transformações de similaridade no modelo de estado ou de restringir as variáveis de otimização envolvidas na recuperação do ganho.

Notação: $M \in \mathbb{R}^{m \times p}$ denota uma matriz real $M$ com $m$ linhas e $p$ colunas. A transposta da matriz $A$ é designada por $A^{\prime}$. Para matrizes simétricas, $X \succ 0$ denota que $X$ é definida positiva, enquanto $X \geq 0$ significa $X=\left[x_{i j}\right], x_{i j} \geq 0, \forall i, j$. O símbolo $\star$ representa um bloco simétrico em uma matriz quadrada.

\section{FUNDAMENTOS DE SISTEMAS POSITIVOS}

Esta seção reúne definições e fundamentos de sistemas positivos necessários para o desenvolvimento dos resultados propostos.

Definição 1. (Farina and Rinaldi (2000)). Um sistema linear é chamado de positivo se estados e saídas do sistema são não negativos para quaisquer estados iniciais e entradas não negativos. Definição 2. (Farina and Rinaldi (2000)). A matriz $A \in \mathbb{R}^{n \times n}$ é chamada de Metzler se todos os elementos fora da diagonal forem não negativos, ou seja, $A_{i j} \geq 0$ para todo $i \neq j$.
Considere o sistema linear contínuo no tempo

$$
\left\{\begin{array}{l}
\dot{x}(t)=A x(t)+B w(t) \\
y(t)=C x(t)+D w(t) .
\end{array}\right.
$$

em que $x(t)$ é o vetor de estados, $y(t)$ são as saídas e $w(t)$ são entradas de distúrbio (não controladas). O sistema (1) é positivo se e somente se $A$ for Metzler, $B \geq 0, C \geq 0$ e $D \geq$ 0 . Adicionalmente, o sistema (1) é estável se as condições apresentadas no lema seguinte forem verificadas.

Lema 1. (Farina and Rinaldi (2000)). O sistema positivo (1) é assintoticamente estável se e somente se existir uma matriz de Lyapunov diagonal definida positiva $P$ tal que

$$
A^{\prime} P+P A \prec 0 .
$$

Note que a estabilidade pode ser garantida com $P$ simétrica e definida positiva, e que a existência de uma matriz de Lyapunov $P$ diagonal satisfazendo (2) assegura propriedades de robustez com respeito a perturbações no estado $x(t)$, um resultado conhecido na literatura como $D$-estabilidade (Kaszkurewicz and Bhaya, 1999; Oliveira and Peres, 2005).

\section{DEFINIÇÃO DO PROBLEMA}

Considere o sistema linear incerto positivo contínuo no tempo

$$
\left\{\begin{array}{l}
\dot{x}(t)=A(\alpha) x(t)+B(\alpha) u(t) \\
y(t)=C(\alpha) x(t)
\end{array}\right.
$$

em que $x(t) \in \mathbb{R}^{n}$ é o vetor de estados, $u(t) \in \mathbb{R}^{m}$ é o vetor de entradas de controle e $y(t) \in \mathbb{R}^{p}$ é o vetor de saídas. As matrizes do sistema são descritas como combinações convexas de $N$ vértices conhecidos, ou seja

$$
(A(\alpha), B(\alpha), C(\alpha))=\sum_{i=1}^{N} \alpha_{i}\left(A_{i}, B_{i}, C_{i}\right), \quad \alpha \in \Lambda
$$

sendo que $\alpha$ é um vetor de parâmetros invariantes no tempo que pertence ao simplex unitário

$$
\Lambda=\left\{\alpha \in \mathbb{R}^{N}: \sum_{i=1}^{N} \alpha_{i}=1, \alpha_{i} \geq 0, i=1, \ldots, N\right\} .
$$

Este artigo aborda o problema de projeto de uma lei de controle robusta por realimentação estática de saída dada por

$$
u(t)=K y(t)
$$

para o sistema (3) tal que o sistema em malha fechada

$$
\dot{x}(t)=(A(\alpha)+B(\alpha) K C(\alpha)) x(t)
$$

seja assintoticamente estável e positivo.

\section{RESULTADOS PRINCIPAIS}

Nesta seção é apresentado o principal resultado deste artigo, que é um procedimento iterativo em termos de condições LMIs dependentes de parâmetros para a síntese de controladores robustos por realimentação estática de saída para sistemas lineares politópicos positivos contínuos no tempo, baseado no teorema a seguir.

Teorema 1. Para uma dada matriz $P_{0}(\alpha)=P_{0}(\alpha)^{\prime} \succ 0$ e um dado escalar real $\mu_{0}(\alpha)>0$, se existirem $P(\alpha)=P(\alpha)^{\prime} \succ 0$, uma matriz $K$, e escalares $\mu(\alpha), \gamma$ e $r$ tais que as seguintes LMIs robustas sejam verificadas para todo $\alpha \in \Lambda$

$$
\left.\left[\begin{array}{c}
-P(\alpha) P_{0}(\alpha)-P_{0}(\alpha) P(\alpha) \\
A_{c l}(\alpha)-r I \\
+\mu_{0}(\alpha) P(\alpha)+\mu(\alpha) P_{0}(\alpha)
\end{array}\right)-2 \mu(\alpha) \mu_{0}(\alpha) I\right] \prec 0,
$$


com $A_{c l}(\alpha)=A(\alpha)+B(\alpha) K C(\alpha)$, então $K$ é um ganho robusto tal que os autovalores de $A_{c l}(\alpha)$ têm parte real à esquerda de $r$ para todo $\alpha \in \Lambda$ e a positividade do sistema (3) em malha fechada é assegurada.

Prova. Primeiramente observe que a desigualdade (9) certifica a positividade em malha fechada do sistema (nenhum termo fora da diagonal é negativo), ou seja, $(A(\alpha)+B(\alpha) K C(\alpha))$ é Metzler. Adicionalmente, note que (8) pode ser rescrita como

com

$$
\mathscr{Q}(\alpha)+\mathscr{X}(\alpha) \mathscr{B}(\alpha)+\mathscr{B}(\alpha)^{\prime} \mathscr{X}(\alpha)^{\prime} \prec 0
$$

$$
\begin{gathered}
\mathscr{Q}(\alpha)=\left[\begin{array}{cc}
0 & \star \\
\left(A_{c l}(\alpha)-r I\right) & 0
\end{array}\right], \\
\mathscr{X}(\alpha)=-\mu(\alpha)\left[\begin{array}{c}
\tilde{P}(\alpha) \\
-I
\end{array}\right], \quad \mathscr{B}(\alpha)=\mu_{0}(\alpha)\left[\begin{array}{c}
\tilde{P}_{0}(\alpha) \\
-I
\end{array}\right]^{\prime},
\end{gathered}
$$

$\tilde{P}_{0}(\alpha)=\mu_{0}(\alpha)^{-1} P_{0}(\alpha)$ e $\tilde{P}(\alpha)=\mu(\alpha)^{-1} P(\alpha)$. Note ainda que o termo $-2 \mu(\alpha) \mu_{0}(\alpha) I$ que aparece na diagonal da desigualdade (8) assegura que $\mu(\alpha)>0, \forall \alpha \in \Lambda$ (uma vez que $\mu_{0}(\alpha)$ é positivo por hipótese), garantindo $\tilde{P}(\alpha) \succ 0$ e $\tilde{P}_{0}(\alpha) \succ 0$.

Multiplicando-se (8) à esquerda por $\mathscr{B}^{\perp}(\alpha)^{\prime}=\left[\begin{array}{ll}I & \left.\tilde{P}_{0}(\alpha)\right] \text { e à }\end{array}\right.$ direita por $\mathscr{B}^{\perp}(\alpha)$, obtém-se

$$
\tilde{P}_{0}(\alpha)\left(A_{c l}(\alpha)-r I\right)+\left(A_{c l}(\alpha)-r I\right)^{\prime} \tilde{P}_{0}(\alpha) \prec 0 .
$$

Por outro lado, multiplicando-se (8) à esquerda por $\mathscr{X}^{\perp}(\alpha)=$

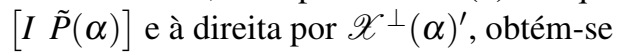

$$
\tilde{P}(\alpha)\left(A_{c l}(\alpha)-r I\right)+\left(A_{c l}(\alpha)-r I\right)^{\prime} \tilde{P}(\alpha) \prec 0 .
$$

As condições (11) e (12) certificam a estabilidade assintótica do sistema com matriz dinâmica dada por $A(\alpha)+B(\alpha) K C(\alpha)-r I$ (reproduzindo uma extensão do Lema 1 para o caso incerto). Isso significa que $K$ é um ganho de realimentação de saída que aloca a parte real dos autovalores do sistema (3) em malha fechada à esquerda de $r$. Se $r \leq 0, K$ é um ganho robusto estabilizante para (3).

O corolário apresentado a seguir é uma adaptação do Teorema 1 para produzir um ganho robusto por realimentação de estados.

Corolário 1. Para uma matriz $P_{0}(\alpha)=P_{0}(\alpha)^{\prime} \succ 0$ e um escalar real $\mu_{0}(\alpha)>0$ dados, se existirem matrizes $P(\alpha)=P(\alpha)^{\prime} \succ 0$, uma matriz $K$, e escalares $\mu(\alpha), \gamma$ e $r$ tais que (8)-(9) sejam verificadas para todo $\alpha \in \Lambda \operatorname{com} A_{c l}(\alpha)=A(\alpha)+B(\alpha) K$, então $K$ é um ganho robusto de realimentação de estados que assegura a positividade e que os autovalores de $A_{c l}(\alpha)$ têm parte real à esquerda de $r, \forall \alpha \in \Lambda$, para o sistema (3) $\operatorname{com} C_{y}(\alpha)=I$.

Note que a condição (8) do Teorema 1 é linear nas variáveis de decisão pelo fato de $\mu_{0}(\alpha)$ e $P_{0}(\alpha)$ serem dados. Para que uma escolha particular de $\mu_{0}(\alpha)$ e $P_{0}(\alpha)$ não seja uma imposição restritiva, foi adicionada uma relaxação de estabilidade ao Teorema 1. Ao invés de alocar os autovalores de malha fechada no semiplano esquerdo, permite-se que os autovalores fiquem alocados em um semiplano à esquerda de $r$ (variável de relaxação), tornando a solução das condições em princípio mais fácil.

O Teorema 1 pode ser usado de maneira recorrente, minimizandose $r$ até obter $r \leq 0$, o que garante a estabilidade do sistema original em malha fechada. Para fazer isso de maneira eficiente, é proposto um algoritmo, cuja principal característica é o fornecimento de soluções factíveis a cada iteração, desde que uma condição inicial adequada seja aplicada simultaneamente a $\mu_{0}(\alpha)$ e $P_{0}(\alpha)$. O resultado a seguir propõe uma escolha que garante a existência de uma solução factível para o Teorema 1.
Teorema 2. A escolha $P_{0}(\alpha)=I$ e $\mu_{0}(\alpha)=1$ garante a existência de uma solução factível para o Teorema 1 .

Prova. Com $P_{0}(\alpha)=I$ e $\mu_{0}(\alpha)=1$, a desigualdade (8) pode ser reescrita como

$$
\left[\begin{array}{c}
-2 P(\alpha) \\
A_{c l}(\alpha)-r I+P(\alpha)+\mu(\alpha) I-2 \mu(\alpha) I
\end{array}\right] \prec 0 .
$$

Escolhendo uma matriz de Lyapunov diagonal, $P(\alpha)=\rho I$, e aplicando-se um complemento de Schur, tem-se

$$
\underbrace{[-2 \mu(\alpha) I]}_{T_{1}}-\underbrace{[\mathscr{M}(\alpha)][-2 \rho I]^{-1}[\mathscr{M}(\alpha)]^{\prime}}_{T_{2}} \prec 0,
$$

em que $\mathscr{M}(\alpha)=A_{c l}(\alpha)-r I+\mu(\alpha) I+\rho I$. Para $\mu(\alpha)>0$, $T_{1} \prec 0$ e se $\rho \rightarrow \infty$, então $T_{2} \rightarrow 0$. Isso significa que para a condição inicial proposta existem pelo menos um $\mu(\alpha)>0$ e $P(\alpha)=\rho I \operatorname{com} \rho$ suficientemente grande tais que (8) seja factível, independentemente do ganho $K$. A escolha $K=0$ garante que (9) também é satisfeita, pois $A(\alpha)$ é por hipótese Metzler.

\subsection{Procedimento iterativo}

Nesta seção apresenta-se a descrição de um procedimento iterativo que resolve as restrições propostas no Teorema $1 \mathrm{e}$, possivelmente, provê um controlador por realimentação estática de saída robustamente estabilizante para o sistema (3). O algoritmo pode ser dividido em duas etapas, que são executadas sequencialmente a cada iteração.

Primeiramente, são inicializadas as variáveis $P_{0}(\alpha)$ e $\mu_{0}(\alpha)$ com os valores definidos pelo projetista (sugere-se $\mu_{0}(\alpha)=1$ e $P_{0}(\alpha)=I$, que sempre produzem solução factível, como mostrado no Teorema 2). Em seguida, inicia-se a etapa 1 do Algoritmo 1, a qual busca uma solução factível para o Teorema 1. Em caso de factibilidade, os valores das variáveis encontradas ( $r, K$ e $\gamma$ ) são armazenados e o algoritmo segue para a etapa 2. Caso contrário, o algoritmo se encerra sem fornecer uma solução estabilizante (a não factibilidade pode ocorrer para condições iniciais diferentes das propostas no Teorema 2).

Na etapa 2, é realizado um teste de sinal na variável $r$, pois $r$ é um limitante superior para a parte real dos autovalores do sistema em malha fechada. Se $r \leq 0$, os autovalores do sistema em malha fechada estão localizados no semiplano esquerdo do plano complexo, indicando que $K$ é um ganho robusto estabilizante e o algoritmo pode ser encerrado.

Por outro lado, se $r>0$, não é possível garantir a estabilidade do sistema. No entanto, uma vez que $r$ é apenas um limitante superior para a parte real dos autovalores do sistema em malha fechada, os autovalores podem ter parte real negativa mesmo com $r>0$. Por esse motivo, quando $r>0$, são realizados testes de análise de estabilidade e positividade do sistema em malha fechada para que, caso a resposta seja factível, o algoritmo possa ser interrompido indicando ter encontrado uma solução estabilizante. Para reduzir o esforço computacional, é feito primeiramente um teste rápido, sequencial, verificando os autovalores dos vértices do politopo $\left(\alpha_{i}=1, \alpha_{j}=0, j \neq i\right) \mathrm{em}$ malha fechada. Se todos forem negativos (o que é uma condição necessária para a estabilidade do politopo), testa-se uma condição LMI de análise de estabilidade e outra de positividade do sistema, e se os resultados confirmarem a estabilidade e positividade, o valor de $K$ armazenado na etapa 1 é um ganho 
estabilizante. Caso contrário, se o maior dos autovalores tiver parte real não negativa ou a condição de análise de estabilidade ou positividade não forem factíveis, os valores de $P(\alpha)$ e $\mu(\alpha)$ encontrados pelo algoritmo nessa iteração, serão os novos valores de $P_{0}(\alpha)$ e $\mu_{0}(\alpha)$ da próxima iteração. Note que a factibilidade está garantida $\left(P_{0}(\alpha)\right.$ e $P(\alpha)$, assim como $\mu_{0}(\alpha)$ e $\mu(\alpha)$ são intercambiáveis em (8)), repetindo-se, no pior dos casos, a solução anterior. Com esta estratégia garantese que o valor de $r$ sempre diminui ou permanece igual ao valor da iteração anterior, mas nunca aumenta. Em outros termos, o algoritmo é localmente convergente.

O procedimento continua até achar um ganho que garanta simultaneamente a positividade e estabilidade assintótica do sistema em malha fechada ( $r \leq 0$, ou solução factível no teste de análise da etapa 2), ou quando o número máximo de iterações $\left(i t_{\max }\right)$ for atingido.

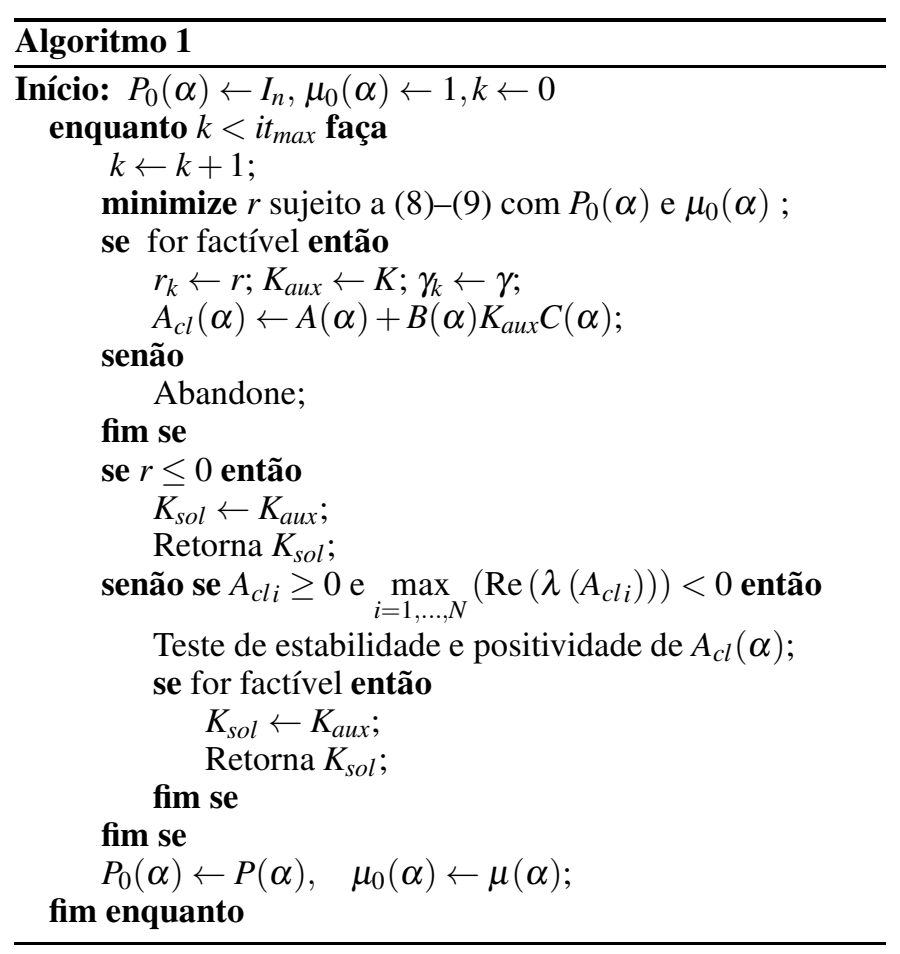

\section{CONSIDERAÇÕES COMPUTACIONAIS}

Antes de apresentar os resultados experimentais deste artigo, é necessário destacar que as condições propostas no Teorema 1 (e Corolário 1) são problemas de dimensão infinita, pois devem ser resolvidos para todo $\alpha \in \Lambda$. Para contornar esse obstáculo, utilizam-se as chamadas relaxações, que são condições suficientes na forma de LMIs construídas a partir da imposição de estruturas polinomiais homogêneas de grau fixo para as variáveis de otimização do problema (Oliveira and Peres, 2007). Esse conjunto finito de LMIs pode ser gerado automaticamente pelo toolbox Robust LMI Parser (ROLMIP) (Agulhari et al., 2019). Particularmente, nos exemplos da seção seguinte, emprega-se grau 1 nas variáveis do Teorema 1 que dependem de $\alpha$. Todos os códigos computacionais foram programados em Matlab (R2014a) empregando os pacotes Yalmip (Löfberg, 2004) e ROLMIP (Agulhari et al., 2019) e os resolvedores Mosek (Andersen and Andersen, 2000) e SeDuMi (Sturm, 1999).

\section{EXPERIMENTOS NUMÉRICOS}

Esta seção apresenta ${ }^{1}$ um experimento numérico para avaliar o desempenho estatístico da abordagem desenvolvida neste artigo no que se refere à síntese de controladores robustos que asseguram a estabilidade e positividade de sistemas incertos em malha fechada. Outro objetivo desta seção é comparar o método de síntese proposto neste artigo com outras técnicas da literatura apresentadas em Bhattacharyya and Patra (2018), (BP18), e Feng et al. (2011), (FLLS11). Deve-se destacar que, como as condições da literatura não tratam o caso incerto, os exemplos comparativos investigam o controle de sistemas positivos precisamente conhecidos usando modelos retirados da literatura ou uma base de sistemas positivos sem incertezas.

\subsection{Estabilização robusta (teste estatístico)}

Pretende-se estabilizar uma base composta por 1000 sistemas incertos (com incertezas politópicas) de $N=2,3,4,5$ vértices, com $n=2,3,4,5$ estados e $m=p=1,2,3,4$ entradas $(m)$ e saídas $(p)$, gerados aleatoriamente. Todos os sistemas da base são positivos e têm solução comprovada (garantidamente estabilizáveis) mas não são quadraticamente estabilizáveis, ou seja, não é possível encontrar solução factível usando uma única matriz de Lyapunov (independente de parâmetros).

São apresentados resultados estatísticos em termos da porcentagem de sistemas estabilizados pelo método proposto ( $\operatorname{Sol}(\%)$ ), o número médio de iterações (It.) e o tempo médio gasto em segundos $(\mathrm{T}(s))$ para resolver cada caso.

Na Tabela 1, são apresentados os resultados obtidos pelo Algoritmo 1 com $i t_{\max }=13$ e com dois resolvedores diferentes: SeDuMi e Mosek. Duas estruturas distintas foram impostas à matriz de Lyapunov $P(\alpha)$ : diagonal (D) ou simétrica (S).

Tabela 1. Resultados obtidos com o Algoritmo 1 para estabilização robusta (teste estatístico).

\begin{tabular}{c|c|c|c|c}
\hline \hline$P(\alpha)$ & Resolvedores & Sol $(\%)$ & It. & $\mathrm{T}(s)$ \\
\hline \multirow{2}{*}{$\mathrm{D}$} & SeDuMi & 77,8 & 4,6 & 1,02 \\
\cline { 2 - 5 } & Mosek & 98,7 & 1,4 & 0,22 \\
\hline \multirow{2}{*}{$\mathrm{S}$} & SeDuMi & 97,8 & 2,5 & 0,79 \\
\cline { 2 - 5 } & Mosek & 99,3 & 1,4 & 0,24 \\
\hline \hline
\end{tabular}

Ao analisar a Tabela 1, pode-se observar que o uso de uma matriz de Lyapunov simétrica fornece resultados melhores que uma estrutura diagonal. Apesar disso, destaca-se que utilizar uma matriz de Lyapunov com mais variáveis não assegura necessariamente melhores resultados estatísticos via procedimento iterativo. O que é possível garantir é que na primeira iteração o resultado (em termos de $r$ ) baseado em uma LMI com matriz de Lyapunov simétrica inclui a estrutura diagonal como caso particular. No entanto, como o procedimento iterativo busca soluções em um espaço não convexo (que tem dimensões diferentes para os casos diagonal e simétrico), o resultado final não pode ser inferido a priori. Como esperado, o tempo médio de cômputo de solução está diretamente relacionado ao número de iterações necessárias para se chegar a uma conclusão, sendo que, se a quantidade de iterações necessárias diminui, o tempo também é menor. É possível verificar também que o resolvedor Mosek requer um número menor de iterações, resultando em

\footnotetext{
1 As bases de dados usadas nos experimentos podem ser encontradas em http://www.dt.fee.unicamp.br/ ricfow/programs/SBAI2019/ baseSistemasPositivosSBA19.zip
} 
tempos computacionais bem menores. Note ainda que, como uma estrutura diagonal utiliza menos variáveis escalares no problema de otimização, isso pode tornar o tempo de resolução de cada iteração menor. Esse fato fica evidente ao comparar a estrutura de $P(\alpha)$ utilizando o resolvedor Mosek, com o mesmo número médio de iterações mas o tempo médio da solução usando $P(\alpha)$ diagonal inferior ao tempo empregando $P(\alpha)$ simétrica.

Finalmente, observa-se que o melhor resultado obtido com o Algoritmo 1 foi com matriz de Lyapunov simétrica e o resolvedor Mosek, chegando a fornecer ganhos estabilizantes para 99,3\% dos sistemas, requerendo em média 1,4 iterações para produzir uma solução em aproximadamente $0,24 \mathrm{~s}$.

\subsection{Exemplos da literatura}

Esta seção tem o objetivo de comparar o Algoritmo 1 usando a matriz de Lyapunov com estrutura simétrica (melhor resultado do Experimento 1) com a técnica de FLLS11 e os métodos apresentados em BP18. Em BP18, são propostos dois algoritmos (chamados de BP18-1 e BP18-2) para estabilizar sistemas precisamente conhecidos lineares invariantes no tempo positivos com múltiplas entradas e múltiplas saídas por realimentação de saída. Os quatro primeiros exemplos (Ex 1, 2, 3, 4) apresentados em BP18 consistem na estabilização de sistemas precisamente conhecidos sem a imposição de estruturas ao ganho. $\mathrm{O}$ quinto exemplo de BP18 propõe a estabilização do sistema investigado no primeiro exemplo (Ex 1) impondo quatro diferentes restrições de estrutura no ganho $K$ (Ex 5.1, 5.2, 5.3, 5.4). O BP18-1 é capaz de prover soluções estabilizantes apenas para três dos quatro primeiros exemplos, e nenhum dos exemplos com restrições. Por outro lado, o BP18-2 provê soluções estabilizantes para todos os exemplos propostos. Nenhum dos demais métodos utilizados nas comparações em BP18 (FLLS11, Shen and Lam (2015), Ait Rami (2011), Wang and Huang (2013)) foi capaz de fornecer soluções factíveis para todos os exemplos. No entanto, o método iterativo descrito no Algoritmo 1 é capaz de prover ganhos de realimentação de saída em todos os casos considerados em BP18.

Para melhor avaliar o conservadorismo dos métodos, considere o projeto de um controlador por realimentação estática de saída para os sistemas dos exemplos de BP18 substituindo a matriz dinâmica $A$ por $A+\rho I$. O objetivo é verificar qual dos métodos é capaz de garantir soluções para uma faixa mais ampla de valores (positivos) de $\rho$. Para este experimento foram testados o Algoritmo 1 proposto neste artigo, os dois algoritmos propostos por BP18 (BP18-1 e BP18-2), e o algoritmo proposto em FLLS11. Todos os algoritmos foram testados com o resolvedor SeDuMi, que fornece os melhores resultados com os métodos da literatura. O número máximo de iterações imposto para todos os algoritmos é $i t_{\max }=20$. A Figura 1 apresenta os resultados para os quatro primeiros exemplos enquanto a Figura 2 mostra os resultados dos exemplos com restrições na estrutura no ganho $K$. Note que o método proposto é melhor em praticamente todos os casos, nunca produzindo resultados piores.

\subsection{Estabilização de sistemas sem incerteza (teste estatístico)}

Pretende-se comparar o desempenho estatístico dos métodos da literatura BP18 e FLLS11 com a abordagem proposta neste artigo na estabilização de uma base (gerada aleatoriamente) composta por 1000 sistemas precisamente conhecidos positivos

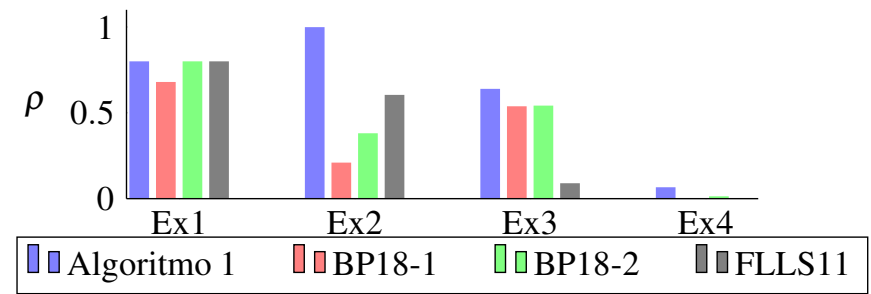

Figura 1. Faixa estabilizável de $\rho$ nos exemplos 1 a 4 de BP18.

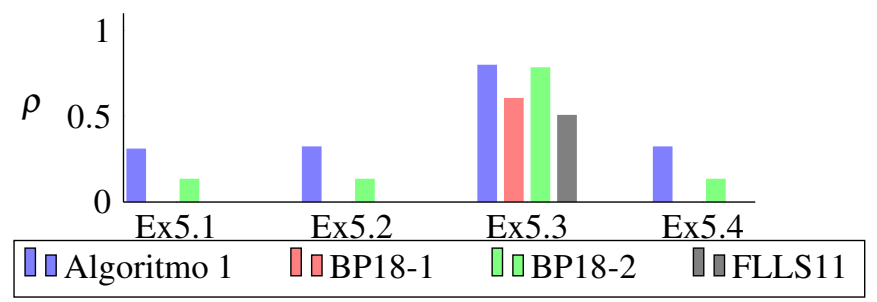

Figura 2. Faixa estabilizável de $\rho$ nos exemplos 5.1 a 5.4 de BP18 (ganho com estrutura).

garantidamente estabilizáveis com $n=2,3,4,5$ estados e $m=$ $p=1,2,3,4$ entradas $(m)$ e saídas $(p)$. Para ser o mais justo possível na comparação, todos os algoritmos foram testados com o resolvedor SeDuMi, o qual forneceu melhores resultados para os métodos da literatura, e um máximo de 20 iterações foi imposto a todos os métodos. A Tabela 2 apresenta as porcentagens de sistemas estabilizados (Sol(\%)), o número médio de iterações (It.) e o tempo médio de cômputo exigido pelos algoritmos $(\mathrm{T}(s))$.

Tabela 2. Resultados obtidos pelo Algoritmo 1 e os métodos BP18-1, BP18-2 e FLLS11 para estabilização da base de sistemas sem incerteza.

\begin{tabular}{l|c|c|c}
\hline \hline Método & Sol $(\%)$ & It. & $\mathrm{T}(s)$ \\
\hline Algoritmo 1 & $94,9 \%$ & 2,4 & 0,42 \\
\hline BP18-1 & $21,6 \%$ & 15,6 & 3,18 \\
\hline BP18-2 & $42,9 \%$ & 13,6 & 1,26 \\
\hline FLLS11 & $5,5 \%$ & 1,9 & 0,15 \\
\hline \hline
\end{tabular}

O Algoritmo 1 obteve resultados bem superiores aos demais, fornecendo um ganho estabilizante para 94,9\% dos sistemas da base. Outro dado interessante é o número de iterações: o Algoritmo 1 precisa de menos de três iterações para resolver a maioria dos problemas, quantidade bem menor que as necessárias para BP18-1 e BP18-2, os quais obtêm menos da metade de soluções estabilizantes que o Algoritmo 1. Finalmente, observe que o algoritmo FLLS11 proposto por Feng et al. (2011), embora consiga resolver o problema de estabilização mais rapidamente, requerendo menos iterações, encontra solução para apenas $5,5 \%$ dos sistemas testados.

\section{CONCLUSÃO}

Este artigo apresentou um método iterativo baseado em LMIs dependentes de parâmetros para prover controladores por realimentação de saída para sistemas incertos positivos. Uma das vantagens do método é a possibilidade de projetar controladores impondo estruturas particulares na matriz do ganho sem restringir as demais variáveis do problema, pois o ganho é tratado diretamente como uma variável de otimização. O baixo conservadorismo do método iterativo quando comparado a outros 
métodos da literatura foi ilustrado por meio de vários experimentos numéricos. Apresentou-se também uma discussão a respeito das estruturas adotadas nas variáveis do problema, dos resolvedores utilizados e do esforço computacional requerido em termos de tempo de solução e número de iterações.

\section{REFERÊNCIAS}

C. M. Agulhari, R. C. L. F. Oliveira, and P. L. D. Peres. LMI relaxations for reduced-order robust $\mathscr{H}_{\infty}$ control of continuoustime uncertain linear systems. IEEE Trans. Autom. Control, 57(6):1532-1537, June 2012.

C. M. Agulhari, A. Felipe, R. C. L. F. Oliveira, and P. L. D. Peres. Algorithm 998: The Robust LMI Parser — A toolbox to construct LMI conditions for uncertain systems. ACM Trans. Math. Softw., 45(3):36:1-36:25, August 2019. http: //rolmip.github.io.

M. Ait Rami. Solvability of static output-feedback stabilization for LTI positive systems. Syst. Control Lett., 60(9):704-708, September 2011.

E. D. Andersen and K. D. Andersen. The MOSEK interior point optimizer for linear programming: An implementation of the homogeneous algorithm. In H. Frenk, K. Roos, T. Terlaky, and S. Zhang, editors, High Performance Optimization, volume 33 of Applied Optimization, pages 197-232. Springer US, 2000. http: //www . mosek. com.

H. Arneson and C. Langbort. A linear programming approach to routing control in networks of constrained linear positive systems. Automatica, 48(5):800-807, May 2012.

A. Berman and R. J. Plemmons. Nonnegative Matrices in The Mathematical Sciences. Computer Science and Applied Mathematics. Academic Press, New York, NY, 1979.

S. Bhattacharyya and S. Patra. Static output-feedback stabilization for MIMO LTI positive systems using LMI-based iterative algorithms. IEEE Control Syst. Letts., 2(2):242-247, April 2018.

S. Boyd, L. El Ghaoui, E. Feron, and V. Balakrishnan. Linear Matrix Inequalities in System and Control Theory. SIAM Studies in Applied Mathematics, Philadelphia, PA, 1994.

C. Briat. Robust stability and stabilization of uncertain linear positive systems via integral linear constraints: $l_{1}$-gain and $l_{\infty}$-gain characterization. Int. J. Robust Nonlinear Control, 23(16):1932-1954, November 2013.

L. Caccetta and V. G. Rumchev. A survey of reachability and controllability for positive linear systems. Ann. Oper. Res., 98(1):101-122, December 2000.

Y. Ebihara, D. Peaucelle, and D. Arzelier. LMI approach to linear positive system analysis and synthesis. Syst. Control Lett., 63:50-56, January 2014.

L. El Ghaoui and S. I. Niculescu, editors. Advances in Linear Matrix Inequality Methods in Control. Advances in Design and Control. SIAM, Philadelphia, PA, 2000.

L. Farina and S. Rinaldi. Positive Linear Systems: Theory and Applications. John Wiley \& Sons, New York, NY, 2000.

A. Felipe. Um algoritmo de busca local baseado em LMIs para computar ganhos de realimentação estabilizantes diretamente como variáveis de otimização. M.Sc. Thesis, FEEC - Universidade Estadual de Campinas, Campinas, SP, Brazil, 2017.
J. Feng, J. Lam, P. Li, and Z. Shu. Decay rate constrained stabilization of positive systems using static output feedback. Int. J. Robust Nonlinear Control, 21(1):44-54, December 2011.

E. Kaszkurewicz and A. Bhaya. Matrix Diagonal Stability in Systems and Computation. Birkhäuser, Boston, MA, 1999.

J. Löfberg. YALMIP: A toolbox for modeling and optimization in MATLAB. In Proc. 2004 IEEE Int. Symp. on Comput. Aided Control Syst. Des., pages 284-289, Taipei, Taiwan, September 2004. http: //yalmip. github . io.

D. G. Luenberger. Introduction to Dynamic Systems. Theory, Models \& Apllications. John Wiley \& Sons, Inc., New York, NY, 1979.

R. C. L. F. Oliveira and P. L. D. Peres. A simple and less conservative test for D-stability. SIAM J. Matrix Anal. Appl., 26(2):415-425, January 2005.

R. C. L. F. Oliveira and P. L. D. Peres. Parameter-dependent LMIs in robust analysis: Characterization of homogeneous polynomially parameter-dependent solutions via LMI relaxations. IEEE Trans. Autom. Control, 52(7):1334-1340, July 2007.

R. C. L. F. Oliveira and P. L. D. Peres. Análise e controle de sistemas lineares por meio de desigualdades matriciais lineares. In A. P. Feltrin, C. R. Minussi, M. C. M. Teixeira, and R. A. R. Lázaro, editors, Tutoriais do XVIII Congresso Brasileiro de Automática, pages 203-229. Cultura Acadêmica, São Paulo, 2010. ISBN 978-85-7983-061-7.

D. Peaucelle and D. Arzelier. An efficient numerical solution for $\mathscr{H}_{2}$ static output feedback synthesis. In Proc. 2001 Eur. Control Conf., pages 3800-3805, Porto, Portugal, September 2001.

B. Roszak and E. J. Davison. Necessary and sufficient conditions for stabilizability of positive LTI systems. Syst. Control Lett., 58(7):474-481, July 2009.

J. Shen and J. Lam. On static output-feedback stabilization for multi-input multi-output positive systems. Int. J. Robust Nonlinear Control, 25(16):3154-3162, November 2015.

J. Shen and J. Lam. Static output-feedback stabilization with optimal $L_{1}$-gain for positive linear systems. Automatica, 63: 248-253, January 2016.

J. Shen and J. Lam. Some extensions on the bounded real lemma for positive systems. IEEE Trans. Autom. Control, 62(6):3034-3038, June 2017.

J. F. Sturm. Using SeDuMi 1.02, a MATLAB toolbox for optimization over symmetric cones. Optim. Method Softw., 11 (1-4):625-653, 1999. http://sedumi.ie. lehigh .edu/.

T. Tanaka and C. Langbort. The bounded real lemma for internally positive systems and $\mathrm{H}$-infinity structured static state feedback. IEEE Trans. Autom. Control, 56(9):22182223, September 2011.

H. S. Vieira, R. C. L. F. Oliveira, and P. L. D. Peres. Robust stabilization and $\mathscr{H}_{\infty}$ control by means of state-feedback for polytopic linear systems using LMIs and scalar searches. In Proc. 2015 Amer. Control Conf., pages 5966-5973, Chicago, IL, USA, July 2015.

C. Wang and T. Huang. Static output feedback control for positive linear continuous-time systems. Int. J. Robust Nonlinear Control, 23(14):1537-1544, September 2013. 